\title{
A five patient's case study on the influence of two different probiotics on individual intestinal microbiota
}

\author{
Yoko Uchiyama-Tanaka
}

Yoko Clinic, 3-3-13 Takami, Yahatahigashi-ku, Kitakyushu 805-0016, Japan

Corresponding address: Yoko Uchiyama-Tanaka, PhD, MD, Yoko Clinic, 3-3-13 Takami, Yahatahigashi-ku, Kitakyushu 805-0016, Japan

Submission date: April 6, 2013; Acceptance date: May 27, 2013; Publication date: May 30, 2013

\begin{abstract}
Background: The composition and activities of indigenous intestinal microbiota are of paramount importance to human immunity, nutrition, and pathological processes, and hence, the health of the individual. It is well established that the intestine is an important site for local immunity. It is known that the effect of probiotics increases beneficial microbiota and improves chronic conditions such as atopic diseases, irritable bowel disease, and obesity. However, as there are so many probiotics, it is unknown which probiotics might have more of an impact upon intestinal microbiota.
\end{abstract}

Objective: To understand how two different types of probiotics influence human intestinal microbiota, we analyzed human fecal microbiota after taking each of the probiotics.

Methods: Five outpatients from Yoko Clinic (1 male and 4 females; aged between 34-46 years old) were enrolled in this study. None of the subjects had cancer or any active inflammatory diseases. The five patients took Lactobacillus buchneri (SU) for 4 weeks, no probiotics the following week, and mixed probiotics (NS) which are Lactobacillus plantarum (NS-5), Lactobacillus rhamnosus (NS-11), Lactobacillus delbruekii (NS-12), Lactobacillus helveticus (NS-8), Lactobacillus fermentum (NS-9) for the following 4 weeks. Fecal samples were collected before and after the outpatients took each of the two probiotics, and were then analyzed using a kit from Techno Suruga Laboratory Co., Ltd. The analysis of the microbiota was performed by targeting bacterial $16 \mathrm{~S}$ rRNA genes with a terminal restriction fragment length polymorphism analysis program (Nagashima method).

Results: Three patients of the five patients decreased the percentage of beneficial bacteria 
(Lactobacillales, Bifidobacteria) after taking SU $(13.7 \pm 7.1 \%$ to $4.0 \pm 3.5 \%)$, whereas the remaining two patients showed an increased percentage of beneficial bacteria (16.8 $\pm 3.4 \%$ to $30.4 \pm 4.6 \%$ ). After taking NS, the three patients who decreased the percentage of beneficial bacteria after taking SU increased their beneficial bacteria $(4.0 \pm 3.5 \%$ to $8.0 \pm 2.1 \%)$, whereas the two patients who increased beneficial bacteria after taking SU showed a decreased percentage of beneficial bacteria $(30.4 \pm 4.6 \%$ to $16.4 \pm 7.4 \%)$.

Conclusion: This study demonstrated that the two probiotics had different influences. Probiotics should be selected not only for their quality but also for compatibility with each intestinal bacterial flora.

Key words: probiotics, intestine, microbiota

\section{BACKGROUND:}

The bacterial count in the intestinal tract is more than $10^{12}$ cells per gram of dry content [1-3], comprising approximately 40-500 species [4]. The composition and activities of indigenous intestinal microbiota are of paramount importance in human immunity, nutrition, pathological processes, and hence, the overall health of an individual [5]. The effect of probiotics increases the count of beneficial microbiota in the intestinal tract and improves chronic conditions, such as atopic diseases, irritable bowel disease, and obesity [6,7]. However, because there are several probiotic species, it is remains unknown which probiotics may have more of an impact upon intestinal microbiota. To understand the influence of two different types of probiotics on human intestinal microbiota, we analyzed human fecal microbiota after supplementation of probiotics.

\section{INTRODUCTION:}

A poor diet and dysregulated digestion can inhibit nutrient bioavailability and absorption, undermine homeostasis, and can create conditions favorable for the onset of disease and metabolic disorders [6,7]. The benefits of balanced intestinal microbiota include maintaining a strong digestive system, enhancing the bioavailability of nutrients to the cells, supporting immune function and cellular viability, and removing metabolic byproducts and environmental toxins.

Each individual has different intestinal microbiota which is depending on the exposure of the fetus to various microbes in the microbes in the birth canal, whether an infant is breast fed, daily diet, environmental and psychological stressors and the indiscriminating use of antibiotics. It has been reported that ethnicity can be a factor influencing different flora [4], and intestinal microbiota of the Japanese includes marine bacteria because of their diet [8]. In a previous experiment, we measured the human intestinal microbiota among different individuals and observed that each person contained different proportions of bacteria [9]. 
Based on these findings, the compatibility of probiotics with an individual's intestinal microbiota content should be considered.

\section{MATERIALS AND METHODS:}

Population. All procedures in this study were performed in accordance with the guidelines of the 2000 Declaration of Helsinki for human experimentation, and all subjects provided signed informed consent. Five outpatients (1 male and 4 females; age, 34-46 years) from Yoko Clinic (Kitakyushu, Japan) were enrolled in this study. The patient characteristics are shown in Table 1 . None of the subjects had cancer or active inflammatory diseases.

Table 1 Clinical characteristics of the study subjects

\begin{tabular}{llll}
\hline Patient & Age & Gender & Underlying disease \\
\hline No. 1 & 47 & Male & Pollen allergy \\
No. 2 & 41 & Female & Asthma \\
No. 3 & 56 & Female & Atopic disease \\
No. 4 & 41 & Female & Constipation \\
No. 5 & 34 & Female & Chronic eruption \\
\hline
\end{tabular}

Materials. In this study, we used two different kinds of probiotics: (1) The first probiotics (Origin Biochemical Inc.) is obtained from cultured rice used in Funa-zushi, which contains Lactopbacillus buchneri (SU), and is consumed as a traditional health food in Japan to prevent the occurrence of colds and indigestion [10]. (2) The second (Laca Co.) probiotics is a mixture of probiotics (NS) such as Lactobacillus plantarum (NS-5), Lactobacillus rhamnosus (NS-11), Lactobacillus delbrueckii (NS-12), Lactobacillus helveticus (NS-8), and Lactobacillus fermentum (NS-9) which are cultured from a grass in Mongolia.

Study Design. All five patients took SU for 4 weeks, no probiotics the following week, and NS for the next 4 weeks. Fecal samples were collected before and after supplementation of each of the two probiotics, and subsequently analyzed using an assay kit from Techno Suruga Laboratory Co., Ltd. No conditions were placed on the patients during this study in terms of dietary intake.

Analysis of Fecal Microbiota. Fecal samples were collected three times (before probiotic supplementation, 4 weeks after SU administration, and 4 weeks after NS administration). Fecal microbiota was analyzed by targeting bacterial 16S rRNA genes using a terminal restriction fragment length polymorphism (T-RFLP) analysis as previously described by Nagashima et al. (11-13). The primers 516F (5'-TGCCAGCAGCCGCGGTA-3') and 1510R (5'-GGTTACCTTGTTACGACTT-3') were used to amplify the 16S rRNA genes. The 5' ends of the 516F forward primer were labeled using 6'-carboxyfluorescein (6-FAM), which was synthesized by Applied Biosystems Japan (Tokyo, Japan). The purified PCR products $(2 \mu \mathrm{L})$ 
were digested with $10 \mathrm{U}$ of $B s l \mathrm{I}$ (New England BioLabs, Inc., Ipswich, USA) at $55^{\circ} \mathrm{C}$ for $3 \mathrm{~h}$. The length of the terminal restriction fragments was determined using an ABI PRISM 3130x1 genetic analyzer (Applied Biosystems, Tokyo, Japan).

\section{RESULTS:}

The percentage of beneficial bacteria decreased in three of the five patients (Lactobacillales, Bifidobacteria) after SU administration (13.7 $\pm 7.1 \%$ vs. $4.0 \pm 3.5 \%)$, but increased in the remaining two patients $(16.8 \pm 3.4 \%$ vs. $30.4 \pm 4.6 \%)$. After NS administration, the percentage of beneficial bacteria increased in the three patients who experienced a decrease in the percentage of beneficial bacteria after SU administration $(4.0 \pm 3.5 \%$ vs. $8.0 \pm 2.1 \%)$, but decreased in the two patients who experienced an increase in beneficial bacteria after SU administration $(30.4 \pm 4.6 \%$ vs. $16.4 \pm 7.4 \%$ ) (Figure 1$)$.

Figure 1. Beneficial fecal bacteria (Lactobacillales and Bifidobacteria) change among prestudy and after taking Lactobacillus buchneri (SU), and mixed probiotics (NS).

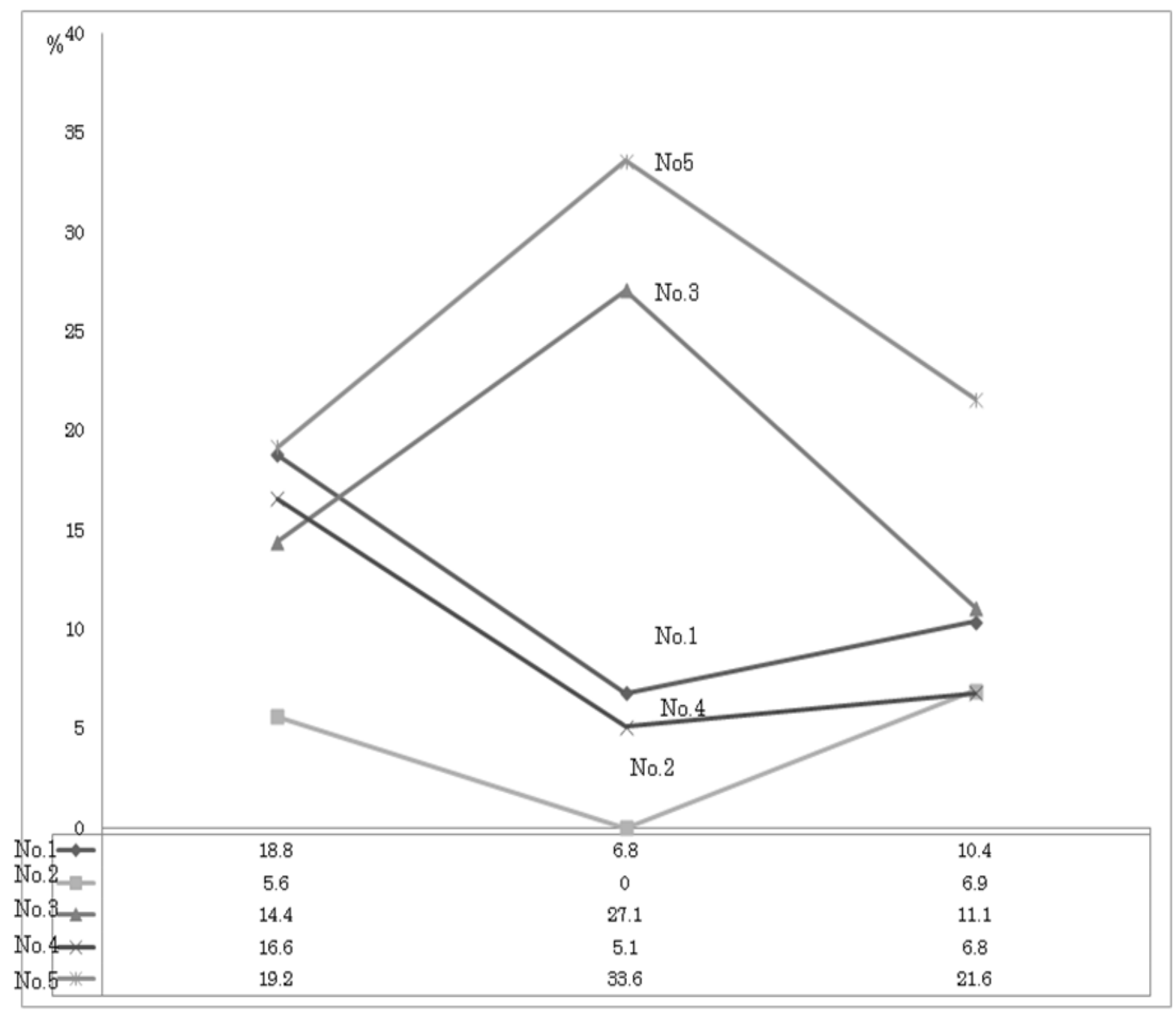

\section{Pre taking probiotics Post taking SU Post taking NS}

Note: No. 1-5 represents individual patient subjects 
According to Collins et al. [14], Clostridium clusters and subclusters cannot divide the unknown that is beneficial or harmful in the intestine. For example, Faecalibacterium prausnitzii is an important butyrate-producing bacterium in Clostridium cluster IV. In contrast, Clostridium perfringens is a well-known harmful bacterium in Clostridium cluster I. Lactobacillales and Bifidobacteria are considered beneficial fecal bacteria. In this study, the beneficial bacterial count decreased in some patients. Clostridium showed no tendency after taking SU and NS either (data not shown).

\section{DISCUSSION:}

A healthy gastrointestinal tract in individuals may not be properly established from birth because of some factors such as the environment inside the mother's uterus and the through birth canal, a diet of breast milk and/or powdered milk, daily diet, as well as exposure to environmental toxins and stressors. Imbalances in intestinal microbiota may induce allergic diseases, metabolic syndrome, diabetes, obesity, immune disorders, and inflammatory bowel disease; however, supplementation of resident microbiota with probiotics may decrease the incidences of these diseases. For example, a recurrent Clostridium difficile infection can be treated by duodenal infusion of donor feces which means changing the recipient's constituent microbiota content by decreasing the proportion of the Clostridium cluster IV and XIVa [15]. Probiotic supplementation is used worldwide to treat allergic diseases, skin disorders, and constipation.

The most important factors for improving the intestinal microbiota are proper well-balanced diet and minimal exposure to environmental and psychological stressors, although probiotic supplementation can also be beneficial. One report showed that the effect of a strict vegetarian diet on the intestinal microbiota was extremely different compared with that of non-vegetarian diet [16]. Probiotic supplementation may highlight the importance of intestinal care in everyday life. However, according to this study, the intestinal microbiota of some patients possibly deteriorated because one probiotic was incompatible. Thus, consumption of incompatible probiotics may be harmful. As previously mentioned, because the intestinal microbiota is different in each person, compatibility of all probiotics should be considered.

There were a few limitations to this study. First, the present study included a small study population, and second, the actual bacterial count was not determined by the method used. T-RFLP was only useful for estimating the bacterial count. Therefore, a more quantitative method for bacterial analysis in addition to the analysis of cost versus performance is required.

In conclusion, this study demonstrated that the two types of probiotics had different effects; therefore, probiotics should be selected not only for their quality but also for compatibility with the host's intestinal microbiota.

Acknowledgements: This research was funded by Origin Biochemical Laboratory Inc. and 
Laca Co.

\section{REFERENCES:}

1. Hayashi H, Sakamoto M, Benno Y. Phylogenetic analysis of human gut microbiota using 16S rDNA clone libraries and strictly anaerobic culture-based methods. Microbiol Immunol 2002, 46: 535-548.

2. Langendijk PS, Schut F, Jansen GJ, Raangs GC, Kamphuius GR, Wilkison MH, Welling GW. Quantitative fluorescence in situ hybridization of Bifidobacterium spp. With genus-specific 16S rRNA-targeted probes and its application in fecal samples. Appl Environ Microbiol 1995, 61: 3069-3075.

3. Suau A, Bonnet R, Sutren M, Godon JJ, Gibson G.R, Collins MD, Dore J. Direct analysis of genes encoding 16S rRNA from complex communities reveals many novel molecular species within the human gut. Appl Environ Microbiol 1999, 65: 4799-4807.

4. Moor WE, Holdeman LV. Human fecal flora: the normal flora of 20 Japanese-Hawaiians. Appl Environ Microbiol. 1974, 27: 961-979.

5. Cerf-Bensussan N, Guy-Grand D, Griscelli C. Intraepithelial lymphocytes of human gut: isolation, characterization and study of natural killer activity. Gut. 1985, 26: 81-88.

6. Rook GA, Brunet LR. Microbes, immunoregulation, and the gut. Gut. 2005, 54(3): 317-320

7. Marik PE. Colonic flora, probiotics, obesity and diabetes. Front Endocrinol. 2012, 3; 87

8. Hehemann J-H, Correc G, Barbeyron T, Helbert W, Czjzek M, Michel G. Transfer of carbohydrate-active enzymes from marine bacteria to Japanese gut microbiota. Nature 2010, 464: 908-914

9. Uchiyama-Tanaka Y. Colon irrigation and lymphocyte movement to peripheral blood. Biochemical Research. 2009,30:311-314.

10. Oshima N, Fujii T. Effect of administered lactobacillus isolated from FunaZushi on fecal micro flora of rats. J Food MIcrobiol 1994, 11(2): 129-132

11. Ando A, Sakata S, Koizumi Y, Mitsuyama K, Fujiyama Y, Benno Y. Terminal restriction fragment length polymorphism analysis of the diversity of fecal microbiota in patients with ulcerative colitis. Inflamm Bowel Dis. 2007, 13: 955-962.

12. Nagashima K, Hisada T, Sato M, Mochizuki J. Application of new primer-enzyme combinations to terminal restriction fragment length polymorphism profiling of bacterial populations in human feces. Appl Environ Microbiol 2002, 69: 1251-1262.

13. Nagashima K, Mochizuki J, Hisada T, Suzuki S, Shimomura K. Phylogenetic analysis of $16 \mathrm{~S}$ ribosomal RNA gene sequences from human fecal microbiota and improved utility of terminal restriction fragment length polymorphism profiling. Bioscience Microflora. 2006, 25: 99-107. 
14. Collins MD, Lawson PA, Willems A, Cordoba JJ, Fernandez-Garayzabal J, Garcia P, Cai J, Hippe H, Farrow JAE. The Phylogeny of the Genus Clostridium: Proposal of Five New Genera and Eleven New Species Combinations. Int J Syst Bacteriol. 1994, 44: 812-826.

15. Nood E, Vrieze A, Niuwdrop M, Fuentes S, Zoetendal EG, de Vos WM, Visser AE, Kujiper EJ, Bartelsman JFWM, Tijissen JGP, SPeelman P, Dijkgraaf MGW, Keller JJ. Duodenal infusion of donor feces for recurrent Clostridium difficile. N Engl J Med 2013, 368(5): 407-415.

16. Hayashi H, Sakamoto M, Bennno Y. Fecal microbial diversity in a strict vegetarian as determined by molecular analysis and cultivation. Microbiol Immunol 2002, 46: 819-831. 